\title{
Incidence, Clinical Manifestation, Treatment Outcome, and Drug Susceptibility Pattern of Nontuberculous Mycobacteria in HIV Patients in Tehran, Iran
}

\author{
Seifu Gizaw Feysia ${ }^{1}$, Malihe Hasan-nejad ${ }^{2,3}$, Siroos Amini $^{4}$, Gholamreza \\ Hamzelou $^{4}$, Hossein Kazemian ${ }^{1,5}$, Jalil Kardan-Yamchi ${ }^{1}$, Morteza Karami- \\ Zarandi $^{1}$, Mohammad Mehdi Feizabadi ${ }^{1,6^{*}}$
}

OPEN ACCESS

Citation: Seifu Gizaw Feysia, Malihe Hasan-nejad, Siroos Amini, Gholamreza Hamzelou, Hossein Kazemian, Jalil Kardan-

Yamchi, Morteza Karami-Zarandi, Mohammad Mehdi Feizabadi. Incidence, Clinical Manifestation, Treatment Outcome, and Drug Susceptibility Pattern of Nontuberculous Mycobacteria in HIV Patients in Tehran, Iran. Ethiop J Health Sci. 2020; 30(1):75. doi:http://dx. doi.org/10.4314/ejhs.v30 i1.10

Received: May 1, 2019

Accepted: August 26, 2019

Published: January 1, 2020

Copyright: (C2020 Seifu Gizaw Feysia, et $a l$. This is an open access article distributed under the terms of the Creative Commons Attribution License, which permits unrestricted use, distribution, and reproduction in any medium, provided the original author and source are credited. Funding: This research was supported by grant from Tehran University of Medical Sciences (Grant No. 38104)

Competing Interests: The authors declare that this manuscript was approved by all authors in its form and that no competing interest exists.

Affiliation and Correspondence:

${ }^{1}$ Department of Microbiology, School of Medicine, Tehran University of Medical Sciences, Tehran, Iran

${ }^{2}$ Department of Infectious Diseases, School of Medicine, Tehran University of Medical Sciences, Tehran, Iran

Iranian Research Center for HIV/AIDS,

${ }^{3}$ Tehran University of Medical Sciences,

Tehran, Iran

${ }^{4}$ Referral Tuberculosis Laboratory, Tehran University of Medical Sciences, Tehran, Iran

${ }^{5}$ Clinical Microbiology Research Center, Ilam University of Medical Sciences, Ilam, Iran

${ }^{6}$ Thoracic Research Center, Tehran University of Medical sciences, Tehran, Iran; *Email: mfeizabadi@tums.ac.ir
ABSTRACT

BACKGROUND: Nontuberculous mycobacterial (NTM) infections have radically increased worldwide due to the increase in HIV infections. The disease activity increases with progressive immunodeficiency.

METHODS: A total of $216 \mathrm{HIV}$ seropositive patients suspected of having mycobacterial infection were recruited for this study. Clinical samples were collected from each patient and cultured on Lowenstein-Jensen media. Detection and species identification were simultaneously done using Reverse Blot Hybridization Assay System. Also, the minimum inhibitory concentrations (MIC) for each isolate were determined in $7 \mathrm{H9}$ broth media for 10 antibiotics.

RESULTS: In this study, 4 rapid and 4 slow-growing NTM species were isolated and identified. Mycobacterium fortuitum was the most common NTM species, 3/8 (37.5\%), followed by Mycobacterium kansasii, 2/8 (25\%). The cases were identified as pulmonary disease, $5 / 8(62.5 \%)$, disseminated infection, $2 / 8$ (25\%), and skin abscess, 1/8 (12.5\%). M. chelonae and Mycobacterium avium were isolated from patients diagnosed with disseminated infection with treatment failure. The skin abscess was caused by infection with $M$. simiae. The results of the MIC testing were as follows: M. kansasii and M. fortuitum were susceptible to amikacin (AMK); M. avium to clarithromycin (CLA); M. fortuitum 2/3 (67\%) to ciprofloxacin (CIP); $1 / 2$ (50\%) of M. kansasii isolates to $C L A$, and $M$. chelonae to rifampin (RIF), linezolid (LIN), AMK, and CIP at medium and high concentrations.

CONCLUSION: AMK showed incredible in vitro activity against M. kansasii and M. fortuitum. Also, M. avium was susceptible to CLA, whereas M. simiae and M. chelonae were resistant to the tested drugs in this study.

KEYWORDS: Nontuberculous mycobacteria; Minimum inhibitory concentrations; Abscess; Microdilution. 


\section{INTRODUCTION}

The relative proportion of mycobacterial infections in HIV patients varies in different geographic areas, indicating the background frequency of latent Mycobacterium tuberculosis (MTB) infections in the local population (1) and the incidence with which NTM are encountered in the environment (2). Studies have identified over 150 NTM species in the world, most of which can cause mycobacterioses in humans $(3,4)$. Recently, infection due to NTM has radically increased worldwide because of HIV infection (4). It can cause pulmonary infection in people living with HIV and may show similar clinical presentations such as fever, cough, weight loss, and night sweat steady with tuberculosis (TB) (5); both show as acid-fast bacilli by microscopy and discrimination needs biochemical or molecular tests (6). The global epidemiology of NTM disease is compromised, because the infections were not reported in most parts of the globe. In Asia, the epidemiological studies of NTM disease have not been population-based. In 2005, a study conducted in Japan revealed that the prevalence of pulmonary NTM was 33 to 65 per 100000 , with the most dominating cases being M. avium complex (7). In a meta-analysis done in Iran in 2015, it was found that the prevalence of NTM among culture positive cases for TB diagnosis was $10.2 \%$, and $M$. kansasii, $M$. simiae, $M$. gordonae, $M$. scrofulaceum, and $M$. chelonae were the most prevalent species (2).

The drug regimen used for treating MTB is usually ineffective in treating NTM, because many of NTMs are naturally resistant to those drugs (4). Therefore, molecular identification is needed to differentiate NTMs at species level and also from MTB complex. Moreover, drug susceptibility testing is essential for selecting effective and appropriate chemotherapy treatment in HIV coinfected patients (8).

Reverse Blot Hybridization Assay System for the identification of mycobacterial species (REBA Myco-ID) is a reliable molecular method for rapid identification of NTM. This method is based on line probe technology and has been designed such that it can simultaneously differentiate 21 MTB and NTM species using PCR, followed by reverse blot hybridization assay. The high sensitivity and specificity with outstanding reproducibility makes this method a highly suitable tool for rapid screening of clinical isolates in high prevalence mycobacteria-HIV coinfection settings.

The aim of this study was to identify the incidence of nontuberculous mycobacteria and the clinical manifestations of the disease. Moreover, it explores the drug susceptibility profile of the NTM species among HIV patients in Tehran province.

\section{MATERIALS AND METHODS}

Study area: This study was conducted in Tehran, the capital city of Iran and Tehran province. It is located in the north of the country, with a population of about 8.7 million in the city and 15 million in the larger metropolitan region of the Greater Tehran. Also, Tehran is the most populated city in Iran and in Western Asia (https://www.amar.org.ir/Portals/1/Iran/90.pdf).

Study population: All HIV seropositive patients presenting for HIV care and treatment services at Emam Khomeini Referral Hospital and 5 health centers in Teheran province who were diagnosed with mycobacterial diseases between April 2018 and July 2019 were recruited for this study. A total of 216 HIV patients identified to have one or more of the classic symptoms of mycobacterial infections such as fever, night sweats, cough, and weight loss were included in the study. The sputum samples were obtained from the patients on spot and the next morning. Other samples such as blood, cerebrospinal fluid (CSF), and stool were also obtained from the patients.

Laboratory tests and culture: Based on the guidelines of the National TB Control Program, 3 sputum samples were collected from each patient. According to the modified Petroff's method, the sputum specimens were homogenized and decontaminated with $4 \% \mathrm{NaOH}$ solution (9). The clinical specimens were examined for the presence of acid-fast bacilli (AFB) by AFB smear. The decontaminated specimens were cultured on solid Lowenstein-Jensen (LJ) and middlebrook 7H9 broth medium. DNA was extracted from the culture positive specimens for molecular analysis. DNA was extracted using DNA extraction solutions (MolecuTechREBA Myco-ID kit), 
according to the manufacturer's manual. Briefly, $50 \mathrm{~mL}$ of the DNA extraction solution was added to the prepared colony in a microtube and vortexed for 1 minute and heated at $100^{\circ} \mathrm{C}$ for 10 minutes using a thermomixer. About 3-5 $\mu \mathrm{L}$ of the supernatant was used as a template for polymerase chain reaction (PCR).

Species identification: In addition to biochemical tests (Niacin and nitrate reduction test) and phenotypic (pigmentation profile and rate of growth) testing, the Reverse Blot Hybridization Assay (REBA) System was used for the detection and identification of $M$. tuberculosis complex and nontuberculous mycobacteria species. The REBA test was based on reverse-hybridization of mycobacterial specific probe and amplified products for rpoB gene. The whole process of REBA was divided into 4 steps: (1) DNA extraction from clinical specimens, (2) multiple PCR amplification of target gene using specific primers, (3) reveres blot hybridization, and (4) color development by chromogenic reaction (colony pigment) (10). PCR was performed on the DNA samples using the methodology described below: predenaturation at $94^{\circ} \mathrm{C}$ one cycle for 5 minutes, (denaturation at $94^{\circ} \mathrm{C}$ for 30 seconds, annealing at $68^{\circ} \mathrm{C}$ for 30 seconds) for 45 cycle, and final elongation at $72^{\circ} \mathrm{C}$ Co one cycle for 7 minutes. The PCR product was used for reverse blot hybridization. The data were interpreted according to the provided data sheet; the marker lane was aligned with the respective lines on the sheet as shown in Figure 1.

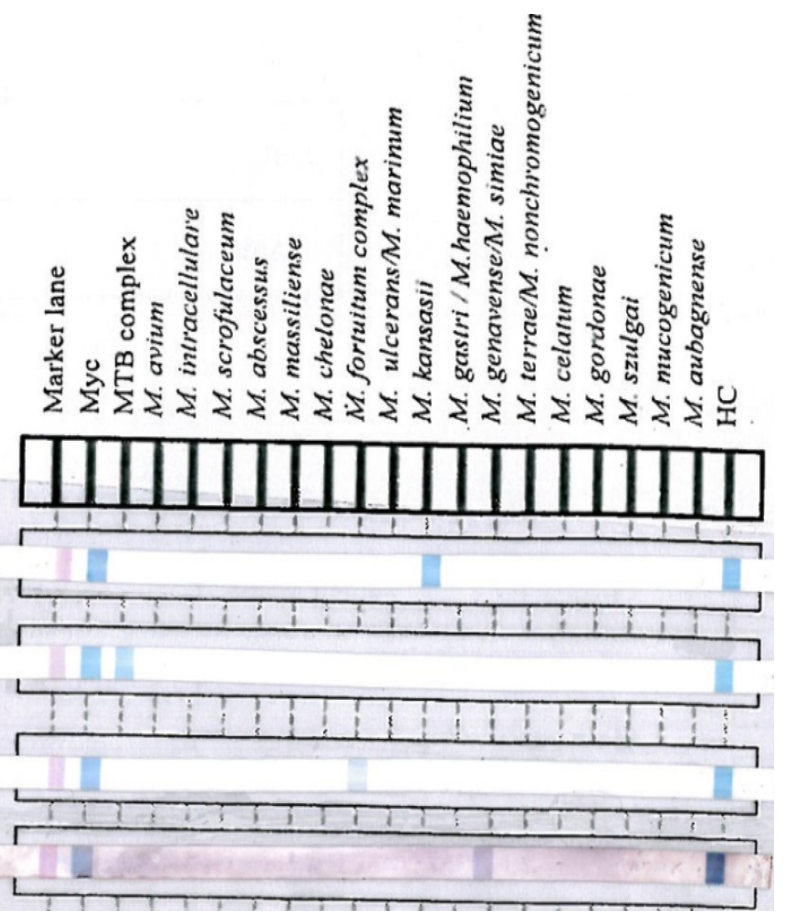

Figure 1: Simultaneous identification of NTM species and MTB complex Myc; cross-reactive of bacteria except mycobacteria, $\boldsymbol{H C}$; Hybridization control

Drug susceptibility testing: The drug susceptibility pattern of NTM was examined using 7H9 broth media for 10 antibiotics, including ethambutol (EMB), rifampin (RIF), isoniazid (INH), amikacin (AMK), clarithromycin (CLA), ciprofloxacin (CIP), imipenem (IMP), linezolid (LIN) cefoxitin (CET), and azitromycin (AZT). The minimum inhibition concentrations (MIC) were established by microdilutions in middlebrook 7H9 broth base on polystyrene 96-well (12 columns and 8 rows) plates based on the Clinical and Laboratory Standards Institute (CLSI) guidelines and published studies. A $50 \mu \mathrm{L}$ of broth media was added to all wells from column 1 to 10 on microplate. For the stock solution of each drug, a final high working concentration was prepared in $1000 \mu \mathrm{L}$ broth medium in separate microtube. A 50 $\mu \mathrm{L}$ of the high working concentration of each drug was added to the corresponding wells on first row from column 1 to 10 , with each well containing 50 $\mu \mathrm{L}$ broth media by using multichannel pipette, serial dilutions were prepared from top to bottom (first row to 8 th row) for each column (1 to 10) by 
transferring $50 \mu \mathrm{L}$ (mix gently for each moves) and finally discarded into 11th column for antibiotic control (such that each row contained 10 drugs, whereas each column contained a single drug). The following final drug concentration ranges were used in the present study: 1 to $128 \mu \mathrm{g} / \mathrm{mL}$ of RIF; 1 to 128 of INH; 0.5 to $64 \mu \mathrm{g} / \mathrm{mL}$ of EMB; 0.5 to 64 $\mu \mathrm{g} / \mathrm{mL}$ of AMK; 0.25 to $32 \mu \mathrm{g} / \mathrm{mL}$ of CIP; 0.5 to $64 \mu \mathrm{g} / \mathrm{mL}$ of CLA; 1 to $128 \mu \mathrm{g} / \mathrm{mL}$ of AZT; 0.5 to $64 \mathrm{of} \mathrm{LIN;} 2$ to 256 of CEF, and 0.5 to $64 \mu \mathrm{g} / \mathrm{mL}$ of IMP. Opacity of the suspension was adjusted to match Mc Farland 1 standard with saline to obtain a stock suspension of approximately $1.5 \times$ 108 colony forming units (CFU)/ml for slowgrowing and $0.5 \mathrm{Mc}$ Farland approximately $0.5 \times$ $107 \mathrm{CFU} / \mathrm{ml}$ rapid-growing NTM. About $100 \mu 1$ of the suspension was transferred to $4900 \mu 1$ (50:5000) of broth media, and then, $50 \mu 1$ of the prepared bacterial suspension was transferred to each well in columns 1 to 10 of the 96 well microplate and sealed in a plastic bag. Subsequently, the microplate was incubated at $37^{\circ} \mathrm{C}$. After the third day of incubation at $37^{\circ} \mathrm{C}$, the MIC of each drug was determined each day up to the last day of the incubation period (the last day of the third week). Detailed information can be found in the study protocol

(https://dx.doi.org/10.17504/protocols.io.nu5dey6).

Data analysis: Data were analyzed using SPSS software. Also, REBA Myco-ID data sheet, provided by the manufacturer, was used to interpret the species identification data.

Ethics statement: The study protocol was approved by the ethics committee of the Vice-

Table 1: Baseline characteristics of sample culture.
Chancellor for Research Affairs in Tehran University of Medical Sciences, (Approval ID: IR.TUMS.UCR.REC.1397.339).

\section{RESULTS}

Patient characteristics and clinical features: In the present study, 216 participants, 57 (26.4\%) females and $159(73.6 \%)$ males, confirmed to be HIV seropositive and suspected of having mycobacterial infections were recruited. The age range of the patients was 14-85 years, with the mean age of 40.14 and standard deviation of 11.42. More than $93 \%$ of the samples collected were sputum and 2\% were CSF. Among the samples, $21 / 216(9.9 \%)$ were smear positive whereas $28 / 216$ $(13 \%)$ were culture positive for mycobacteria (NTM and MTB complex) (Table 1); 8/216 (3.7\%) of the sample were positive for NTM species. The NTM species isolated in the present study included: slow-growing species, Mycobacterium kansasii, Mycobacterium. simiae, and Mycobacterium avium, and the rapid-growing species, Mycobacterium fortuitum, and Mycobacterium chelonae. Among the 8 NTM isolates, $4 / 8(50 \%)$ were rapid-growing species, and the others $4 / 8 \quad(50 \%)$ were slow-growing. According to the biochemical test in the present study, only M. simiea was positive for niacin test, whereas $M$. kansasii and $M$. fortiutum were positive for nitrate test. Moreover, only the colony of M. kansasii cultured on LJ changed color to yellow 48 hours after light exposure, but none of the other cultures showed color change.

\begin{tabular}{lcc}
\hline & Culture Negative N (\%) & Culture positive N (\%) \\
\cline { 1 - 2 } Demographic & & 38.73 \\
Age (year) & 40.33 & $3(5.3 \%)$ \\
Female & $54(94.7 \%)$ & $25(15.7 \%)$ \\
Male & $134(84.3 \%)$ & \\
\hline Specimen & & $23(11.5 \%)$ \\
\hline Sputum & $177(88.5 \%)$ & $1(25 \%)$ \\
Blood & $3(75 \%)$ & $1(6.7 \%)$ \\
CFS & $5(83.3 \%)$ & $1(25 \%)$ \\
Pleural aspiration & $3(75 \%)$ & $1(50 \%)$ \\
Stool & $1(50 \%)$ & $1(100 \%)$ \\
Abscess & - & \\
\hline
\end{tabular}

DOI: http://dx.doi.org/10.4314/ejhs.v30i1.10 
The male to female ratio of the patients in this study was $7: 1$. Pulmonary diseases were observed in $5 / 8(62.5 \%)$ patients who were tested for NTM positive species. The disseminated infections caused by $M$. chelonae and $M$. avium were seen in $2 / 8(25 \%)$ of the cases. Similar clinical manifestations were observed including productive cough, shortness of breath, bronchiectasis, and emphysema. Night sweating and chest pain were the main manifestations of disseminated infection. One case $(1 / 8)$ was a local infection caused by $M$. simiae, isolated from a skin abscess of a 28-year old man who presented with low-grade fever (Table 2). Also, M. chelonae was isolated from a hemophilic 34-year old female patient with disseminated infection; blood, CSF, bone marrow, and sputum samples were smear and culture-positive in this patient. The patient had been treated for one year extensively with first and second-line antituberculosis medications, including RFM, INH, EMB, pyrazinamaid, and AMK, based on WHO recommendation for TB treatment. $M$. avium was also similarly isolated from a 36-year old man diagnosed with disseminated infection. Smear and culture of bone marrow aspirate, blood, CFS, sputum, and stool samples were positive for this species in the patient. This patient was admitted to the hospital very late at the critical stage of the disease. Both species isolated from the patients with disseminated infections were resistant to the recommended dosage used for treating $\mathrm{TB}$ patients.

Table 2: The demographic data of patients and NTM by category

\begin{tabular}{lccc}
\hline Variables & N (\%) & Unknown outcome N (\%) & Poor outcome N (\%) \\
\hline NTM cases & 8 & $6(75)$ & $2(25)$ \\
Female & 1 & 0 & $1(100)$ \\
Male & $7(87.5)$ & $6(75)$ & $1(12.5)$ \\
NTM location & & & \\
Pulmonary & $5(62.5)$ & $5(100)$ & 0 \\
Extra-pulmonary & $3(37.5)$ & $1(33.3)$ & $2(66.7)$ \\
Growth rate & & & $1(20)$ \\
Rapid growing & $4(50 \%)$ & $1(20)$ & $1(33.3)$ \\
Slow growing & $4(50 \%)$ & $2(66.7)$ & 0 \\
Microbiology & & & 0 \\
M. kansasii & $2(25)$ & $2(100)$ & $1(100)$ \\
M. simiae & $1(12.5)$ & $1(100)$ & $1(100)$ \\
M. avium & $1(12.5)$ & 0 & 0 \\
M. chelonae & $1(12.5)$ & 0 & \\
M. fortuitum & $3(37.5)$ & $3(100)$ & \\
\hline
\end{tabular}

Species identification: Among the 28 culturepositive isolates, $8 / 28(28.5 \%)$ were identified as NTM isolates using biochemical test, growth rate, and pigmentation profile. Furthermore, the Reverse Blot Hybridization Assay System kit was used for species identification.

Phenotypic drug susceptibility patterns of the NTMs isolates: The MIC values of $M$. kansasii, $M$. fortuitum, M. avium, M. simiae, and $M$. chelonae are presented in Table 3. The pattern of drug susceptibility for the NTM isolates varied between the species and strains. M. kansasii and M. avium complex were more susceptible to RIF, AMK, and CLA from the tested drugs. M. fortiutum was more susceptible to CIP, CAL, and LIN; however, the susceptibility of $M$. chelonae to RIF, AMK, CIP, and LIN was at a higher level of concentration. None of the NTM isolates was susceptible to INH, and IMP at any antibiotic concentration range. Only one isolate belonging to 
M. kansasii was susceptible to $\mathrm{EMB}$ at $\mathrm{MIC}=\geq 8$, whereas the remaining isolates, $7 / 8 \quad(87.5 \%)$, showed resistance to EMB even at the highest concentration. All the rapid- and slow-growing
NTM isolates, $8 / 8(100 \%)$, were resistant to INH and IMP at the highest concentration and both groups showed high susceptibility to RIF and AMK.

Table 3: Comparative drug susceptibility patterns of NTM isolates (N (\%)).

\begin{tabular}{|c|c|c|c|c|c|c|}
\hline Drug & $\begin{array}{l}\text { MIC } \\
(\mu \mathrm{g} / \mathrm{mL})\end{array}$ & $\begin{array}{l}\text { M. } \\
\text { simiae }\end{array}$ & $\begin{array}{l}\text { M. } \\
\text { kansasii }\end{array}$ & $\begin{array}{l}\text { M. } \\
\text { fortuitum }\end{array}$ & $\begin{array}{l}\text { M. } \\
\text { avium }\end{array}$ & $\begin{array}{l}\text { M. } \\
\text { chelonae }\end{array}$ \\
\hline Isoniazid (INH) & $>128$ & $1(100)$ & $2(100)$ & $3(100)$ & $1(100)$ & $1(100)$ \\
\hline \multirow[t]{5}{*}{ Rifampin (RIF) } & 2 & $1(100)$ & & & & \\
\hline & 8 & & $1(50)$ & & $1(100)$ & \\
\hline & 32 & & $1(50)$ & $1(33.3)$ & & \\
\hline & 64 & & & $1(33.3)$ & & $1(100)$ \\
\hline & $\geq 128$ & & & $1(33.3)$ & & \\
\hline \multirow[t]{3}{*}{ Ethambutol (EMB) } & 8 & & $1(50)$ & & & \\
\hline & 32 & & & $1(33.3)$ & & \\
\hline & $>64$ & $1(100)$ & $1(50)$ & $2(66.7)$ & $1(100)$ & $1(100)$ \\
\hline \multirow[t]{5}{*}{ Amikacin (AMK) } & 4 & & $1(50)$ & & & \\
\hline & 8 & & & $1(33.3)$ & & \\
\hline & 16 & & $1(50)$ & & $1(100)$ & \\
\hline & 32 & & & $2(66.7)$ & & $1(100)$ \\
\hline & $>64$ & $1(100)$ & & & & \\
\hline \multirow[t]{5}{*}{ Ciprofloxacin (CIP) } & 2 & & & $1(33.3)$ & & \\
\hline & 4 & & & $1(33.3)$ & & \\
\hline & 8 & $1(100)$ & & & & \\
\hline & 16 & & & $1(33.3)$ & $1(100)$ & $1(100)$ \\
\hline & $>32$ & & $2(100)$ & & & \\
\hline Clarithromycin & 2 & & $1(50)$ & & & \\
\hline \multirow[t]{3}{*}{ (CLA) } & 4 & & & & $1(100)$ & \\
\hline & 16 & & $1(50)$ & & & \\
\hline & $>64$ & $1(100)$ & & $3(100)$ & & $1(100)$ \\
\hline \multirow[t]{2}{*}{ Cefoxitin (CEF) } & 128 & & & & $1(100)$ & \\
\hline & $>256$ & $1(100)$ & $2(100)$ & $3(100)$ & & $1(100)$ \\
\hline \multirow[t]{2}{*}{ Linezolid (LIN) } & 32 & & & $3(100)$ & $1(100)$ & $1(100)$ \\
\hline & $>64$ & $1(100)$ & $2(100)$ & & & \\
\hline \multirow[t]{3}{*}{ Azithromycin (AZT) } & 16 & & & $2(66.7)$ & & \\
\hline & 32 & & & $1(33.3)$ & & \\
\hline & $>128$ & $1(100)$ & $2(100)$ & & $1(100)$ & $1(100)$ \\
\hline Imipenem (IMP) & $>64$ & $1(100)$ & $2(100)$ & $3(100)$ & $1(100)$ & $1(100)$ \\
\hline
\end{tabular}

\section{DISCUSSION}

A number of Mycobacterium species other than MTB have also been identified as human pathogens $(11,12)$. NTM and MTB are 2 different pathogens with similar epidemiological and clinical features, which are difficult to differentiate in resource limited settings. The standard medications used for MTB treatment are ineffective against NTM (12). Consequently, it is important to increase the efficiency and accuracy of diagnostic tools for the diagnosis of NTM and MTB infections to initiate the appropriate medication $(12,13)$. The treatment of NTM depends on the type of species and individual strains. Therefore, early 
identification is critical for the treatment of the disease (14). In the recent century, biochemical tests have been replaced by molecular methods for the characterization of new species, and NTM detection at a subspecies level, which is extensively helpful in facilitating treatments $(15,16)$. It has been suggested that sputum samples alone may not be sufficient for the diagnosis of NTM infections. The symptoms are also variable, including chronic cough with or without sputum production and chest pain due to pulmonary infection (17).

To the best of the knowledge of the authors, the present study is the first to investigate the incidence of NTM-HIV coinfection in Tehran, Iran. A total of $216 \mathrm{HIV}$ patients diagnosed with coinfection with mycobacteria were included in this study. Molecular assays were used for the simultaneous identification of NTM species and MTB complex. In addition to traditional biochemical identification, REBA Myco-ID molecular diagnostic kit was used for the detection and identification of NTM species (Figure 1). The use of phenotypic characters such as pigment production (chromogenic) and rate of growth, together with biochemical tests such as niacin production and nitrate reduction tests may be adequate for the identification of some of the clinically relevant mycobacterial species. However, phenotypic characters and biochemical analysis might occasionally fail to reach ultimate identification (18). In this study, 5 species of NTMs were identified (M. kansasii, M. fortuitum, $M$. avium, $M$. chelonae, and $M$. simiae). Also, $M$. fortiutum (3 isolates) and M. kansasii (2 isolates) were the most prevalent species. This result is similar to that of previous studies in Iran, even though they were conducted on different population groups (19). In the present study, patients with Pulmonary diseases 5/8 (62.5\%) had similar clinical manifestations even though the causative agents belonged to different species. Also, 2/8 (25\%) patients with disseminated infection mainly presented with chest pain, but the patient who was diagnosed with local skin abscess, $1 / 8$ (12.5\%), had no significant symptom. Many evidences have shown that MTB infections overlap with NTM infections, indicating that smear, and culture-positive TB cases may also be positive for
NTM cases. Because of the considerable overlap of clinical signs and symptoms such as emphysema, bronchiectasis and lung cavitation, and other chest x-ray appearance of MTB and NTM lung disease, it is mandatory to identify the organism in order to provide appropriate treatment for the patients with effective medications (18). If undetected, this overlap may be reported as drug-resistant MTB, even though the resistance was due to NTM species. Moreover, the high rate of NTM is probable due to treatment failure (12).

The results of this study showed that the incidence of NTM to MTB complex in the HIV coinfected patients was 1:2.5 in Tehran province; $8 / 28(28.6 \%)$ of culture positive samples diagnosed with MTB). A research study conducted in Iran found that out of 117 isolates suspected of MDR$\mathrm{TB}$, about 30\% were misdiagnosed as MDR-TB, although further molecular identification revealed NTMs, which are resistant to anti-TB drugs. In addition, M. kansasii was the most prevalent species in their study $(12,20)$.

The research study conducted on all TBinfected patients in Nigeria reported that out of the $16.5 \%$ of sputum isolates and culture-positive cases considered to be MTB, upon molecular identification, $4 \%$ were identified as NTM infections (21). On the other hand, a study published in 2013, which assessed the prevalence of NTM in Nigeria, found that out of 1603 alleged TB cases, $15 \%$ were identified as NTM infections (22).

The epidemiological studies of NTM diseases have not been population-based in Asia. A study conducted in Japan reported a prevalence of 33 to 65 per 100000 for NTM, although M. avium complex was the most prevalent in the region. In contrast, a study conducted in Southeast Asia indicated that out of 1060 HIV-infected individuals, $223(21 \%)$ were NTM positive, and only $19(2 \%)$ had NTM disease of whom 9 were pulmonary cases (7). The results of their study are consistent with those of the present study in prevalence, but in their studies, the occurrence of pulmonary cases was less than the present study.

The criteria for drug susceptibility testing for NTM have been established by the Clinical and Laboratory Standards Institute (CLSI). The CLSI 
also recommends drug susceptibility methods and breakpoints for drugs used for treating NTM diseases (23). It has been proven that there is dissimilarity among individual NTM strains towards the susceptibility of an individual drug. Each strain needs its own treatment that should be decided on the basis of the outcome of drug susceptibility tests $(4,23)$. Nevertheless, no molecular assay has been developed which is able to replace culture-based susceptibility method in mycobacteriology laboratory; nor is there an available molecular method for confirming the presence of resistance gene. Therefore, the CLSI guidelines recommends broth microdilution method as the gold standard method for determining the drug susceptibility pattern of NTMs $(4,18)$.

The results of this study are consistent with that reported by other research studies on MIC and numbers (\%) of resistant strains. However, few other studies obtained results which are different from that of the present study (24). Based on the CLSI guidelines, the MIC for each isolate of NTM was determined (Table 3). In this study, it was found that imipenem and isoniazid were less effective against NTM isolates. M. simiae was isolated from a local skin abscess in a 28-year old male patient. This species was less resistant to RIF and CIP, but showed high resistance to other tested drugs. It was the only isolate with the highest MIC value (MIC $>64 \mu \mathrm{g} / \mathrm{mL}$ ) against amikacin, while the other isolates had less MIC values. This isolate was also resistant to the entire drugs included in this study. The $M$. chelonae isolate was susceptible to RIF, AMK, CIP, and LIN at high concentrations, but it was resistant to all other tested drugs even at high concentrations. The patient infected with this bacterium had been extensively treated for 1 year in the hospital with RIF, INH, EMB, and pyrazinamide for 2 months, followed by INH and RIF for 4 months. However, blood, sputum, and CFS smear remained positive. Then, the attending physicians switched to a second-line drug, amikacin, to treat the patient. Since the isolate was resistance at the standard dosage for the treatment of TB, the treatment outcome was poor, which resulted in the death of the patient. Another patient infected with $M$. avium with a highly disseminated infection involving the bone marrow, presented late to the hospital, and unfortunately passed away before the culture result was obtained. The susceptibility of the isolate depends on concentration. At medium concentration, it is susceptible to RIF and AMK. However, it's susceptible only to CLA while the susceptibility to LIN was at higher concentration ( $\geq 32 \mu \mathrm{g} / \mathrm{mL}$ ). The MIC for EMB, $\mathrm{CEF}$, and AZT were higher than the concentration range used in this study. A previous study reported that the MIC values of most $M$. avium complex (MAC) isolates were beyond the doses suggested by pharmacokinetics for the treatment of the disease, particularly with EMB, RIF, and LIN (25). Moreover, research studies have indicated that the drug susceptibility pattern of MAC varies from region to region in the world (26). In this study, 2 of the patients with pulmonary disease were infected with $M$. kansasii, and the isolates produced yellow pigments as the culture was exposed to light. All M. kansasii isolates were susceptible to AMK, and only one (50\%) isolate was susceptible to CLA and EMB. These isolates were susceptible to RIF at high concentration range and resistance to INH, CEF, LIN, IMP, and AZT at the highest concentration range (Table 3). A study conducted in Taiwan found that of the total $M$. kansasii isolates, $27 \%$ were resistant to $\mathrm{INH}$, $21.6 \%$ to RFM, $73 \%$ to $\mathrm{EMB}$, and $2.7 \%$ to $\mathrm{AMK}$, but no resistance to CLA was identified (24). A case study reported that INH was ineffective for the treatment of $M$. kansasii in HIV-infected patient (27). Also, research studies conducted in Iran, Brazil, and Taiwan confirmed that M. kansasii isolates were susceptible to CLA (4). On the other hand, a study conducted in Taiwan in 2009 reported that all $M$. kansasii isolates were susceptible to LIN, which disagrees with the finding of this study (24). All M. fortiutum isolates were susceptible to AMK, similar to M. kansasii isolates, even though the 2 groups had different growth rates. The present study found that $2 / 3$ (66.6\%) of $M$. fortiutum isolates were susceptible to CIP, and all the isolates $(3 / 3)$ were resistant to the remaining tested drugs. In the recent year, a research study reported from China indicated that all $M$. fortiutum (17/17) isolates were extremely resistant to RIF, INH, and EMB (28), which is 
consistent with the results of this study. Other previous studies reported that $\mathrm{M}$. fortuitum isolates were susceptible to CIP and AMK at variable MIC ranges. On the other hand, except for RIF and CIP, M. simiae showed a high resistance rate to AMK, CLA, IMP, CEF, LIN, INH, and EMB.

In this study, the treatment outcome for the 6 patients was not obtained, because they were either lost to follow-up or moved to other provinces. Of the 8 patients referred to the TB laboratory, only 2 underwent anti-TB first-line drug susceptibility test. The remaining patients underwent only smear and culture testing based on the requests from the hospital and health centers. We suspect that those patients treated with first-line antituberculosis drugs were infected with isolates that were resistant to the anti-TB medications, which most probably resulted in treatment failure. The most significant limitation of this study is that the prevalence of NTM-HIV infections was relatively low in Tehran province; therefore, it is difficult to generalize the drug susceptibility patterns for other NTM strains globally. It is difficult to distinguish pulmonary tuberculosis from NTM lung diseases. Also, effective drugs for treating NTM have not yet been developed and standardized. Therefore, differential diagnosis of pulmonary TB from NTM lung disease is critical, and should be followed by determining MIC for the recommended drugs to treat HIV-mycobacteria coinfection. Molecular identification, culture, and determining MIC for individual isolates are the gold standard for facilitating rapid and effective treatment in NTMHIV coinfected patients. In the present study, AMK showed incredible in vitro activity against $M$. kansasii and M. fortuitum. However, M. avium was resistant to the entire tested drugs, except for CLA. Based on the results of this study, we recommend updating the decisions on NTM treatment through periodic and long-term assessment of radiological changes, symptoms, and mycobacterial culture results. Moreover, effective communication among laboratories, clinicians, and radiologists leads to an optimized outcome in the disease management though population-based studies are needed for epidemiological understanding.

\section{ACKNOWLEDGEMENTS}

This study was supported by Tehran University of Medical Sciences (Project No. 38104)

\section{REFERENCES}

1. Nishiuchi $\mathrm{Y}$, Iwamoto $\mathrm{T}$, Maruyama $\mathrm{F}$. Infection sources of a common non-tuberculous mycobacterial pathogen, Mycobacterium avium complex. Frontiers in medicine. 2017;4:27.

2. Nasiri MJ, Dabiri H, Darban-Sarokhalil D, Shahraki AH. Prevalence of non-tuberculosis mycobacterial infections among tuberculosis suspects in Iran: systematic review and metaanalysis. PloS one. 2015;10(6):e0129073.

3. Johnson MM, Odell JA. Nontuberculous mycobacterial pulmonary infections. Journal of thoracic disease. 2014;6(3):210.

4. Litvinov V, Makarova M, Galkina K, Khachaturiants E, Krasnova M, Guntupova L, et al. Drug susceptibility testing of slowly growing non-tuberculous mycobacteria using slomyco test-system. PloS one. 2018;13(9):e0203108.

5. Agizew T, Basotli J, Alexander H, Boyd R, Letsibogo G, Auld A, et al. Higher-thanexpected prevalence of non-tuberculous mycobacteria in HIV setting in Botswana: Implications for diagnostic algorithms using Xpert MTB/RIF assay. PLoS One. 2017;12(12):e0189981.

6. McCarthy KD, Cain KP, Winthrop KL, Udomsantisuk N, Lan NT, Sar B, et al. Nontuberculous mycobacterial disease in patients with HIV in Southeast Asia. American journal of respiratory and critical care medicine. 2012;185(9):981-8.

7. Stout JE, Koh W-J, Yew WW. Update on pulmonary disease due to non-tuberculous mycobacteria. International Journal of Infectious Diseases. 2016;45:123-34.

8. Burke RM, Coronel J, Moore D. Minimum inhibitory concentration distributions for firstand second-line antimicrobials against Mycobacterium tuberculosis. Journal of medical microbiology. 2017;66(7):1023-6.

9. Tripathi K, Tripathi PC, Nema S, Shrivastava AK, Dwiwedi K, Dhanvijay AK. Modified Petroff's method: an excellent simplified decontamination technique in comparison with Petroff's method. Int J Recent Trends Sci Tech. 2014;10(3):461-4. 
10. Wang HY, Kim H, Kim S, Bang H, Kim DK, Lee $H$. Evaluation of PCR-reverse blot hybridization assay for the differentiation and identification of $\mathrm{M}$ ycobacterium species in liquid cultures. Journal of applied microbiology. 2015;118(1):142-51.

11. Dufour A. Pathogenic mycobacteria in water: a guide to public health consequences, monitoring and management: World Health Organization; 2004.

12. Raju RM, Raju SM, Zhao Y, Rubin EJ. Leveraging advances in tuberculosis diagnosis and treatment to address nontuberculous mycobacterial disease. Emerging infectious diseases. 2016;22(3):365.

13. Sarro YD, Kone B, Diarra B, Kumar A, Kodio O, Fofana DB, et al. Simultaneous diagnosis of tuberculous and non-tuberculous mycobacterial diseases: Time for a better patient management. Clinical microbiology and infectious diseases. 2018;3(3).

14. Sebastian G, Nagaraja SB, Vishwanatha T, Hemalatha K, Vijayalakshmi N, Kumar P. Identification of Non-Tuberculous Mycobacterium by LPA (CM/AS) assay, HPLC and biochemical test: which is feasible for RNTCP? Indian Journal of Tuberculosis. 2018;65(4):329-34.

15. Ryu YJ, Koh W-J, Daley CL. Diagnosis and treatment of nontuberculous mycobacterial lung disease: clinicians' perspectives. Tuberculosis and respiratory diseases. 2016;79(2):74-84.

16. Lan R, Yang C, Lan L, Ou J, Qiao K, Liu F, et al. Mycobacterium tuberculosis and nontuberculous mycobacteria isolates from HIVinfected patients in Guangxi, China. The International Journal of Tuberculosis and Lung Disease. 2011;15(12):1669-75.

17. Kwon YS, Koh W-J. Diagnosis of pulmonary tuberculosis and nontuberculous mycobacterial lung disease in Korea. Tuberculosis and respiratory diseases. 2014;77(1):1-5.

18. Adikaram CP. Overview of Non Tuberculosis Mycobacterial Lung Diseases. Mycobacterium: Research and Development. 2018:257.

19. Velayati AA, Rahideh S, Nezhad ZD, Farnia P, Mirsaeidi M. Nontuberculous mycobacteria in Middle East: current situation and future challenges. International journal of mycobacteriology. 2015;4(1):7-17.
20. Shahraki AH, Heidarieh P, Bostanabad SZ, Khosravi AD, Hashemzadeh M, Khandan S, et al. "Multidrug-resistant tuberculosis" may be nontuberculous mycobacteria. European journal of internal medicine. 2015;26(4):27984.

21. Pokam BT, Asuquo AE. Acid-fast bacilli other than mycobacteria in tuberculosis patients receiving directly observed therapy short course in cross river state, Nigeria. Tuberculosis research and treatment. 2012;2012.

22. Aliyu G, El-Kamary SS, Abimiku Al, Brown C, Tracy K, Hungerford L, et al. Prevalence of non-tuberculous mycobacterial infections among tuberculosis suspects in Nigeria. PloS one. 2013;8(5):e63170.

23. Brown-Elliott BA, Nash KA, Wallace RJ. Antimicrobial susceptibility testing, drug resistance mechanisms, and therapy of infections with nontuberculous mycobacteria. Clinical microbiology reviews. 2012;25(3):54582.

24. Wu T-S, Leu H-S, Chiu C-H, Lee M-H, Chiang $\mathrm{P}-\mathrm{C}, \mathrm{Wu} \mathrm{T}-\mathrm{L}$, et al. Clinical manifestations, antibiotic susceptibility and molecular analysis of Mycobacterium kansasii isolates from a university hospital in Taiwan. Journal of antimicrobial chemotherapy. 2009;64(3):511-4.

25. Schön T, Chryssanthou E. Minimum inhibitory concentration distributions for Mycobacterium avium complex - towards evidence-based susceptibility breakpoints. International Journal of Infectious Diseases. 2017;55:122-4.

26. Cho EH, Huh HJ, Song DJ, Moon SM, Lee S$\mathrm{H}$, Shin SY, et al. Differences in drug susceptibility pattern between Mycobacterium avium and Mycobacterium intracellulare isolated in respiratory specimens. Journal of Infection and Chemotherapy. 2018;24(4):315-8.

27. Despotovic A, Savic B, Salemovic D, Ranin J, Jevtovic D. Isoniazid-resistant Mycobacterium kansasii in an HIV-positive patient, and possible development of immune reconstitution inflammatory syndrome after initiation of highly active antiretroviral therapy: case report. International Journal of Infectious Diseases. 2016;42:40-2.

28. Shen Y, Wang X, Jin J, Wu J, Zhang X, Chen J, et al. In Vitro Susceptibility of Mycobacterium abscessus and Mycobacterium fortuitum Isolates to 30 Antibiotics. BioMed research international. 2018;2018. 